\title{
Politik für Schlüsseltechnologien
}

\author{
Entwicklung und Gestaltung von Schlüsseltechnologien ist auch eine Aufgabe \\ der Politik. Die sorgfältige Abstimmung und Nutzung von Forschungs-, Wissen- \\ schafts-, Innovations- und Bildungspolitik sind grundlegende Voraussetzung, \\ um technologischen Wandel übergreifend und gewinnbringend zu gestalten. Oh- \\ ne politisch geschaffene Rahmenbedingungen und strategische Steverung droht \\ das Potential von Schlüsseltechnologien einseitig oder unzureichend ausge- \\ schöpft zu werden.
}

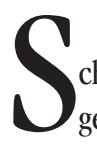
1 Von Edelgard Bulmahn chlüsseltechnologien werden ihrem Namen gerecht. In ihnen steckt großes Potenzial für unsere Forschung, für die Wirtschaft und unsere Gesellschaft. Schlüsseltechnologien schaffen die Voraussetzung für zukunftssichere Beschäftigungen. Und das ist eines der Hauptanliegen unseres politischen Handelns. Vorrangig die Forschungspolitik kann neue Wachstumskräfte mobilisieren und den Weg zu zukunftssicheren Arbeitsplätzen aufzeigen. Der Schlüssel hierzu liegt in einer konsequenten Ausrichtung der Forschungsförderung auf Innovationen. Forschung macht aus Geld Wissen - Innovationen machen aus Wissen Geld.

Innovationen und mit ihnen die Schlüsseltechnologien sind im Zeitalter der Globalisierung der Lebensnerv einer sich entwickelnden Volkswirtschaft. Die Informations- und Kommunikationstechnologie, die Bio- und Nanotechnologie, Mikrosystemtechnik, Produktions-, Werkstoff- und optische Technologien - sie sind die Basis für eine wirtschaftliche Dynamik. Sie sichern unserer Gesellschaft Wachstum, Beschäftigung und Wohlstand.

\section{- Forschungspolitik stößt Innovationen an}

Noch Anfang der 80er Jahre war Deutschland bei der Produktion von Laserstrahlenquellen Entwicklungsland; heute halten deutsche Unternehmen einen Weltmarktanteil von 40 Prozent. Im Jahr 1987 waren 7.000 Personen mit der Herstellung, der Produktion und der Anwendung von Laserstrahlquellen beschäftigt - im Jahr 2001 waren es 60.000 .

Seit 1999 hält Deutschland in Europa den Spitzenplatz bei der Anzahl der Unternehmen, deren Kerngeschäft die Biotechnologie ist. Mit knapp
15.000 Beschäftigten arbeiteten im Jahr 200166 Prozent mehr Menschen in jungen Unternehmen der deutschen Biotechnologiebranche als noch ein Jahr zuvor.

Mit dem ,300-mm-Projekt“ wurde in Deutschland die Grundlage für die Chipfabrik der Zukunft geschaffen. Durch staatliche Förderung im Bereich der Halbleiterindustrie sind in Deutschland insgesamt 16.000 hochwertige Arbeitsplätze geschaffen worden, 11.000 davon allein in der Region Dresden.

Mehr als die Hälfte der Industrieprodukte und über 80 Prozent der Exporte Deutschlands hängen heute vom Einsatz moderner elektronischer Systeme, der Informations- oder Kommunikationstechnik ab. Für den Maschinenbau liefern sie Steuerungen, Test- und Prüfeinrichtungen, in der Chemischen Industrie regeln sie Verfahrensabläufe, in Kraftfahrzeugen bestimmen sie mittlerweile alle wichtigen Funktionen - Antrieb, Kommunikation, Komfort, Sicherheit. Ohne hochleistungsfähige Werkstoffe wären elektronische Systeme undenkbar. Ebenso bei der Datenverarbeitung: Wir stellen mittlerweile derart hohe Ansprüche an die Erfassung und den Transport von Daten, dass dies nur unter Einsatz modernster Werkstoffe, wie Polymere, photonischer Materialien, Funktions- oder Piezokeramik funktioniert.

Mit Exportquoten von teilweise über 50 Prozent sind es gerade die Wirtschaftszweige der Schlüsseltechnologien, mit deren innovativen Produkten Deutschland auf den Weltmärkten Geld verdient. Rund 3,7 Millionen Menschen finden in Unternehmen der Informations- und Kommunikationstechnik, der Chemischen Industrie, der Elektrotechnik/Elektronik und des Maschinenbaus Beschäftigung. Damit hängen mittlerweile über 50 Prozent der Bruttowertschöpfung und der Ar- beitsplätze des Verarbeitenden Gewerbes mit der Entwicklung von Schlüsseltechnologien zusammen. Diese Branchen decken 84 Prozent der FuEAufwendungen und 80 Prozent des FuE-Personals in Deutschland ab.

Die Forschungs- und Innovationspolitik der Bundesregierung hat zu dieser Entwicklung maßgeblich beigetragen. Ein hohes staatliches Engagement für Forschung und Entwicklung bewirkte, dass auch die Forschungsaufwendungen der Wirtschaft stark stiegen. In erfolgreichen Biotechnologie-Regionen kamen auf einen Euro staatliche Förderung bis zu zehn Euro an privaten Mitteln. Der Anteil von Forschung und Entwicklung am Bruttoinlandsprodukt ist wieder auf 2,5 Prozent gestiegen.

Forschungsergebnisse allein schaffen noch keine Arbeitsplätze. Notwendig ist, dass gut ausgebildete Menschen die Forschungsergebnisse umsetzen. Dabei hängen Bildung, Forschung und Beschäftigung kausal zusammen. Deshalb ist die Bildungspolitik als Grundlage der Innovation Deutschlands einer der zentralen politischen Schwerpunkte.

\section{Technologische Kompetenzen für die Wissensgesellschaft}

Den Weg in eine Wirtschaft, die Wissen und Kompetenz zu ihren wichtigsten Ressourcen macht, wollen wir beschleunigen. Es beginnt in der Schule, die das Interesse für technologische Vorgänge weckt, und setzt sich in den beruflichen Lebensläufen fort. Individuelle Kompetenzen gilt es zu entdecken, zu fördern und in die entsprechenden Berufsfelder einzubringen. Die Zukunft der Innovationskompetenz entscheidet sich an unseren Schulen und Hochschulen:

- An den Schulen werden die Grundlagen der naturwissenschaftlich-technischen Kompetenz gelegt und die Aufgeschlossenheit für naturwissenschaftlich-technische Sachverhalte geweckt. Mit dem Programm ,Zukunft Bildung und Betreuung" geben wir wesentliche Impulse, um die Qualität der Bildung in Deutschland zu verbessern. Wir unterstützen daher die Einrichtung zusätzlicher Ganztagsangebote an den Schulen sowie die Einführung nationaler Bildungs- und Leistungsstandards.

- Wir werden die Schaffung neuer, moderner Berufe und die Neuordnung von Ausbildungsberufen weiter vorantreiben.

- Die Tendenz des gestiegenen Interesses für die technischen Studiengänge muss sich weiter fortsetzen. Die Bachelor- und Master-Studiengänge bieten hier besonderes Potenzial. Wir werden mit 
dem „Pakt für Hochschulen“ unseren Beitrag insbesondere für bessere Studienbedingungen, eine stärkere, auch internationale Profilbildung der Hochschulen sowie eine überzeugende Nachwuchsförderung leisten.

Innovationskompetenz - und mit ihr der Einsatz von Schlüsseltechnologien - hat auch eine gesellschaftliche Dimension: Innovationen müssen nicht nur entstehen, sie müssen sich auch durchsetzen. Hierbei spielen oft nicht nur rationale Fragen eine Rolle, wie beispielsweise bei der Elektrosmogdiskussion zu sehen ist. Es geht um Hoffnungen und Ängste. Deshalb darf sich Forschung und Forschungspolitik nicht auf Fragen beschränken, die bereits die öffentliche Diskussion bestimmen. Sie ist gefordert, wie ein Pfadfinder mit den Mitteln der Wissenschaft schleichende Umweltgefährdungen und potenzielle Konfliktlagen aufzuspüren und auf die politische Agenda zu setzen. In einer komplexen, dynamischen Welt voller Unsicherheit über die Zukunft muss die Forschung nachhaltige Strategien zum Umgang mit nicht prognostizierbaren Entwicklungen erarbeiten.

\section{- Risikoforschung ist zentral}

Dabei richtet sich die Forschungsperspektive insbesondere auf die Chancen und Risiken, die mit Schlüsseltechnologien und dem Wirtschaften in vernetzten Systemen einhergehen. Beschleunigte Innovationszyklen ziehen Veränderungen privater und beruflicher Alltagsroutinen jedes Einzelnen bis hin zu einer Neuorientierung ganzer Bereiche gesellschaftlichen Handelns nach sich. Dieser Prozess eröffnet gleichzeitig Chancen und Zeitfenster um einen Wandel in Richtung Nachhaltigkeit zu initiieren.

Methoden der Innovations- und Technikanalyse (ITA) kommen dabei eine besondere Bedeutung zu. Mit diesem Konzept fördert das Bundesministerium für Bildung und Forschung (BMBF) gesellschaftliche Diskurse, in die auch die Visionen und Bedürfnisse der Menschen in den Innovationsprozess einfließen. Aufgezeigt werden soll, welche Fortschritte gesellschaftlich erwünscht sind. Insgesamt soll die ITA in einer hoch technisierten Gesellschaft Orientierung bieten und einen Beitrag zur Förderung einer menschen- und umweltverträglichen Gestaltung von (Schlüssel-) Technologien leisten. So können mit risikoreiche Entwicklungen chancenreichen Alternativen gegenüberstellt und dadurch Möglichkeiten für Nachhaltigkeit und Wettbewerbsfähigkeit geschaffen werden.
Nachhaltigkeitsforschung greift diese Ansätze auf und führt sie weiter, zum Beispiel indem sie soziale und politische Strukturen mit berücksichtigt und fragt, welche Interessenkonstellationen und Akteursbündnisse aussichtsreich sind, um chancenreiche, nachhaltigere Entwicklungen gegenüber riskanten durchsetzen. Hier setzt das BMBF mit dem Förderschwerpunkt ,Sozial-ökologische Forschung“" (SÖF) Maßstäbe - auch im europäischen Vergleich. Im Rahmen von SÖF fördern wir die praxisorientierte, transdisziplinäre Erforschung von gesellschaftlichen Problemlagen und dem zugehörigen komplexen Beziehungsmuster zwischen Gesellschaft und Natur. Dafür arbeiten Geistes- und Naturwissenschaften in Projekten mit Akteuren aus der Praxis zusammen. Ziel ist es, auf der Grundlage des Wissens über die Systeme Umwelt, Wirtschaft und Gesellschaft gesellschaftliche Prozesse so zu beeinflussen, dass sie im „Korridor" einer nachhaltigen Entwicklung bleiben. Ein Weg dabei ist die gezielte Entwicklung und Förderung technischer oder sozialer Innovationen. Derzeit ist eine Bekanntmachung in Vorbereitung, mit der Verbundvorhaben zum Thema ,Schlüsseltechnologien und sozial-ökologischer Wandel“" (TESOWA) gefördert werden sollen. In ihr wird die gegenseitige Beeinflussung von technologischer Entwicklung und gesellschaftlichem Wandel thematisiert.

\section{- Politik muss Dialoge fördern}

Für die Politik besteht die Aufgabe darin, den Dialog zwischen den Systemen zu stärken. Wirtschaft und Wissenschaft sind in den Frühphasen von Technologien oft noch nicht hinreichend abgestimmt. Der Wissenschaft fehlt der Blick auf die kommenden Marktpotenziale. Der Wirtschaft fehlt der Blick für die Notwendigkeit der grundlegenden Forschung beispielsweise für die Bereitstellung von Risikokapital. Dies erschwert im Übrigen auch die politische Entscheidungsfindung. Häufig wird nicht rechtzeitig ein Konsens zwischen Wirtschaft und Wissenschaft erzielt. Manchmal muss am Ende eine Entscheidung gefällt werden, die nicht optimal ist.

Neuere Untersuchungen zeigen, dass in vielen deutschen Betrieben, vor allem in kleineren und mittleren Unternehmen, die Kompetenz zur Umsetzung von Wissen in Innovation deutlich ausgebaut werden kann. Dies betrifft vor allem das fachliche Wissen über Schlüsseltechnologien der Mitarbeiterinnen und Mitarbeiter. Nach Berufseintritt veraltet es - deshalb muss es im Sinne eines lebenslangen Lernens ständig aufge-

\section{Partizipation und Un- ternebmen}

und

\section{Transitionsstrategien \\ zu einer nachbaltigen Wirtschaft}

\author{
sind die Themen \\ der Informationsdienste
}

\section{Ökologisches Wirtschaften 1/04 und 2/04}
Wenn Sie potenzielle Beiträge haben, wenden Sie sich bitte an die Redaktion.

frischt werden. Dies ist in der Regel nur schwer am Arbeitsplatz selbst möglich. Es bedarf vorbereitender und begleitender Aus- und Weiterbildungsmaßnahmen. Mitarbeiterinnen und Mitarbeiter müssen heutzutage unter hohem Arbeitsund Wettbewerbsdruck externes Wissen aufnehmen, komplexe Innovationsprobleme analysieren und Lösungen entwickeln.

Deutschland konnte in den vergangenen Jahren seine Position als einer der weltweit wichtigen Innovationsstandorte behaupten. Nach wie vor hat Deutschland ein weltweit anerkanntes Bildungssystem. Wir verfügen über eine gute Infrastruktur und eine hervorragende Forschungslandschaft. Die Politik der Bundesregierung wird dazu beitragen, dass Deutschland auch in $\mathrm{Zu}$ kunft ein attraktives High-Tech-Land bleibt und über Forschung neue Wachstumskräfte mobilisiert werden.

\section{Die Autorin}

Edelgard Bulmahn ist Bundesministerin für Bildung und Forschung.

Kontakt: Bundesministerium für Bildung und Forschung (BMBF), Hannoversche Str. 28-30, 10115 Berlin. Tel. 01888-575050, Fax 01888-57-5551, E-Mail: presse@bmbf.bund.de 
(c) 20I0 Authors; licensee IÖW and oekom verlag. This is an article distributed under the terms of the Creative Commons Attribution Non-Commercial No Derivates License (http://creativecommons.org/licenses/by-nc-nd/3.o/), which permits unrestricted use, distribution, and reproduction in any medium, provided the original work is properly cited. 